\title{
Safely Connected: Continuous Design for a Mutating City
}

\section{Laura Daglio}

Politecnico di Milano

laura.daglio@polimi.it

ORCID 0000-0002-0645-1094

\section{Elena Mussinelli}

Politecnico di Milano

elena.mussinelli@polimi.it

ORCID 0000-0002-4521-522X

\author{
Daniele Fanzini \\ Politecnico di Milano \\ daniele.fanzini@polimi.it \\ ORCID 0000-0002-4432-6171
}

Irina Rotaru

Ville de Saint-Germain-en-Laye

irina.rotaru@saintgermainenlaye.fr

\begin{abstract}
The renewed interest in the quality of public space is fostered by the need to regenerate parts of the modern city and to improve environmental conditions of urban centres. Major changes due to the recent economic crises, to ICT technologies and to the current pandemic are posing new challenges to the rethinking of the design approach in terms of mobility, health, safety, and communal life. This paper aims at highlighting a renewed attitude to the design of public spaces which reinterprets these issues, also indicating a possible scaling up to other urban contexts. Accordingly, it presents the experience of a recently completed international research project aimed at transforming a French city centre into an open-air urban market. Done through the pedestrianization of streets and encouraging multimodality and multifunctionality of spaces and services, it simultaneously enhances the ability to interpret the needs of the inhabitants and leverages their sense of community.
\end{abstract}

\section{Keywords}

Public space

Co-design

Health

Mobility

E-commerce 


\section{Change, city, public space}

Over the past decades, a renewed interest in the quality and design of public space emerged both due to the need to regenerate parts of the modern city, and from an awareness that the improvement of the environmental conditions of urban centres and the pursuit of sustainable objectives also rest in a substantial rethink of the open spaces (Losasso, 2017, Mussinelli, 2018, Battisti et al., 2020). Revisiting a design approach with these aims must, however, consider the important changes which have altered the relation between the physical space and the wellbeing of its inhabitants. Although on the one hand, the recent economic crises have slowed down traditional urban renewal processes due to a reduction in investment, on the other, ICT technology and social media now offer inhabitants ample access to the content continually produced by the city. This may also present opportunities to acquire new skills, to exchange ideas and forge networks, as well as the creation of new goods and services, produced as a result of the proactive formulas stimulated by the change (Celaschi et al., 2019). The spread of temporary urbanism, has now become a support tool for the urban space modernization strategies (Camocini et al., 2020), providing concrete evidence, firstly, of the possibility of involving citizens in the design process as 'experts of their life experiences'; renewing the modelling of space and thus establishing new rules for the organisation of urban facilities (Gianfrate \& Longo, 2017). These experiences have proved successful both as the result of a unified plan, and as an aggregate of specific actions capable of responding to sectoral areas such as mobility, accessibility, integration of technologies, etc. (Boeri et al, 2018), making a significant contribution to the regeneration of entire urban areas, especially when addressing the real needs of the communities (Gianfrate \& Longo, 2017; Mallach, 2006). Secondly, they demonstrated the capacity of the physical space to accept new uses, diverse densities and ways of interaction, new formal and informal expressions of community living, without losing the recognizable character of the place, but protecting the established heritage and generating new one at the same time (PPS, 2018). The co-design approaches are capable of fostering a sense of belonging to the area and reactivating ways to reclaim public spaces as communal assets (Manzini, 2015; Schiaffonati, 2016), both as a redefinition of the already existing common identity, and as an enlargement of the open spaces use aimed at active mobility, reducing areas of traffic and parking and enhancing environmental quality (GDCl \& Nacto, 2016).

The recent pandemic has a significant impact on this change, a pretext to accelerate the mobility transformation processes already underway. New requirements have also emerged in terms of health, safety, and communal life which also offer a novel perspective on modes of use and the substantial removal of the borders between public and private, also in terms of the redefinition of the functions on an urban, building and dwelling scale. Accordingly, these multiple applications requiring the review of the design processes for the rethinking of the public spaces, offer considerably more than a decorative addition. Advances in digital technology, a renewed responsibility and the potential of the stakeholders 
should also be considered, as well as the multifunctionality and adaptability of the applications, assessing the role of the project as a way to facilitate the physical processes of transformation and reappropriation, aimed at the integration of the material infrastructures with the virtual ones.

Through the experience of a recently completed research in which these new applications linked to the design of the public space are all combined, this study aims to bring to light aspects of a renewed attitude to the project which reinterprets these new applications, also to be replicated in other urban contexts.

\section{The Safely Connected Project: Goals and Methodology}

Safely Connected ${ }^{1}$ was developed by the French Municipality of Saint Germain-en-Laye (SGL) in collaboration with researchers in different disciplines of the ABC Department of the Politecnico di Milano with the involvement of the association of local retailers (CAP SGL) and Fondazione Politecnico. It represents a logical advancement sustained by the continuous efforts and political support of SGL in the field of urban mobility and public space and its progressive evolution in the arena of European projects. By capitalising on the first insights of the ongoing Space4People European network $^{2}$ and following the URBACT method $^{3}$, the municipality seized the opportunity to address the longstanding needs of the local community, which were confirmed through a recent survey on the revival of the city centre commercial area, threatened by the aggressive superstore developments on the outskirts of town.

The project aimed at transforming the city centre of SGL into an open-air urban market by encouraging multimodality and multifunctionality of spaces and services while simultaneously enhancing the ability to interpret the needs of the inhabitants and leveraging their sense of community.

Aimed at experimenting new ways of urban resilience for citizens and retailers affected by the pandemic, the research addressed the complexity of the challenges, with the manifold direct and indirect implications. It developed an integrated approach involving multiple disciplines, stakeholders and tools and providing a flexible organisation in order to manage the constricted timeline defined by the programme.

In fact, from the very beginning, the ambition was to support everybody in dealing with the pandemic context, while following the overall goals and political principles of the city and of the newly installed local elected team. Furthermore, the focus was on comprehensive solutions enabling cities to address complex unexpected situations beyond Covid. Therefore, the project had to simultaneously respond to a large variety of (sometimes opposing) needs while favouring the easy replication, scale-up and scaledown of the validated solutions.
1

https://www.eiturbanmobility.eu/projects/ safely-connected-sustainable-common-accessibility-of-lively-downtowns-for-healthy-people/

$$
2
$$

URBACT network gathering 9 European cities working together on urban mobility issues.

\section{3}

The URBACT Method enables the integrated urban development based on 3 core principles: integration, action learning and participation. https://wwww.google.com/ search?q=the+urbac$\mathrm{t}+$ method\& $\mathrm{rlz}=1 \mathrm{C} 1 \mathrm{CH}-$ BF_enR0763R0763\&o$q=$ the+urbact+meth od\&aqs $=$ chrome..69i 57j69i61.5366j0j7\&sourceid=chrome\&ie $=$ UTF-8\#kpvalbx= JFOYY NjsBsCljLsPilGs 6 Ao17 
Accordingly, the project methodology was configured as a co-design activity based on the following fundamental principles:

- continued involvement of the stakeholders, a condition that allows the public to make their own decisions and protect the common infrastructure.

- dynamic involvement of the stakeholders, i.e.: the solution for an innovation process based on the objectives set by the progress of the program.

The two principles resulted from the belief that public design should produce not only useful artefacts, but also the means for discussion, improvement, and the future autonomy of the communities involved (Teli et al., 2015). Moreover, in order to advance innovative design solutions, it is possible to benefit from well-developed processes to foresee the needs of the people and the nature of contexts within which those innovations fit (Casoni \& Fanzini, 2011; Freire et al. 2017). In this sense, the public space project guided by social innovation has helped the community of retailers and inhabitants of SGL to identify and build their own action strategies.

Furthermore, sustainability is guaranteed through the collaborative and multidisciplinary growth of a common long-term vision prepared and supported by both a short-term and a medium-term plan, designed from an interdisciplinary perspective.

Indeed, the implementation timeline of the project Fig. 1 required specific attention because of the tight deadlines imposed, at the same time dealing with uncertainty due to the emergency context.

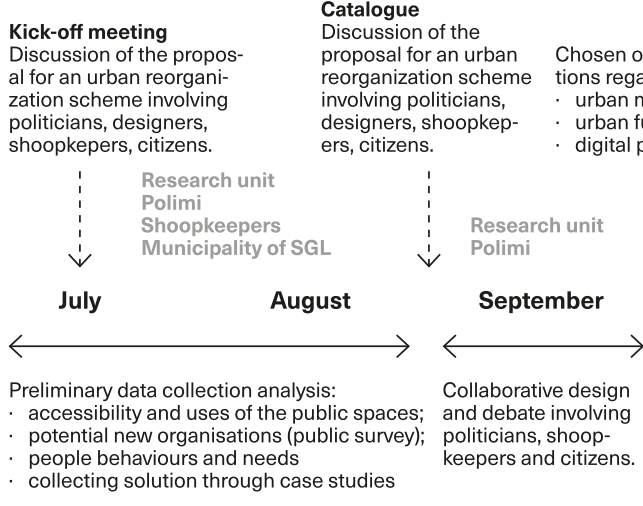

Research unit Polimi Municipality of SGL
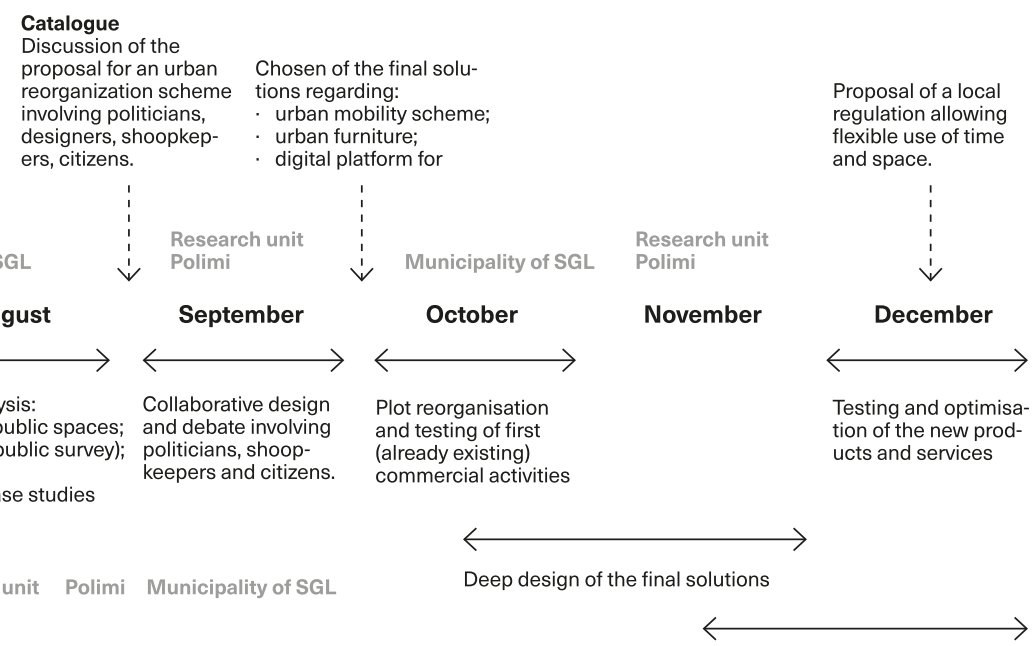

production and installation of the urban furniture prototypes integration of the e-commerce platform (pick-up and delivery goods)

Fig. 1

Project timeline. 
The stakeholder engagement adhered to the following sequence Fig. 2:

1 sense making (hypothesis) consisting of a pre-planning endeavour that prepared the creative activity according to the characteristics of the context and the expectations of the interlocutors. Beginning with problem setting followed by brainstorming, this phase aimed at producing the basic principles of the design activity;

2 pre-design (analysis) corresponding to the construction of a simplified model of reality implemented through the prior definition of a solid and shared vision;

3 conceptualisation of the solution (synthesis) implemented through demonstration projects to stimulate the interest of the stakeholders and generate a process of adaptation and production of new initiatives in the experimental exploration of a vision;

4 testing and adaptation of the prototype for collecting information on how it worked and how to improve it.

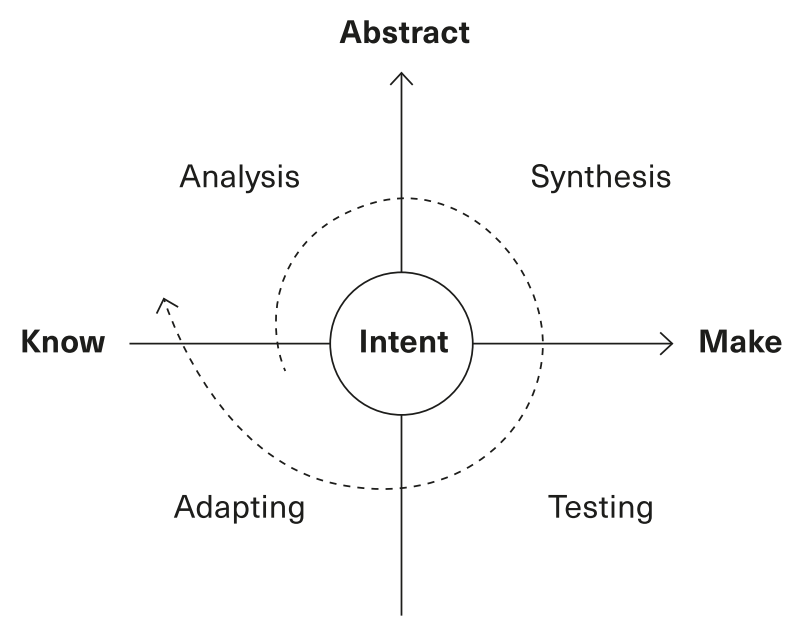

Real

The methodology envisaged the concept and proposed solutions not only to be supported by local input (complaints, suggestions, brief needs assessment etc.) but also to be informed by international best practices outlining the state of the art in the related fields. The co-design activity that involved local administrators, the Association of the Shopkeepers and the SGL Maison de Projets exploited three-dimensional models set within the experimental contexts with the aim of allowing for a direct experience of the design choices. The same solution was used on a larger scale at the end of the process to test the quality of the final result.

Finally, in order to augment the inclusiveness target, and improve the resilience and flexibility in time and space, the physical interventions in the public space were complemented by digital space actions (the active mobility and community friendly e-platform). The latter was conceived in order to include functions that pave the way 
for the adoption of new business models and the optimisation of existing ones, enabling rapid economic, social, and cultural revival.

Safely Connected was exceptional not only from the methodological point of view, but also at the level of EIT Urban Mobility, the focus of which is large metropolises. SGL is the only small sized city that has been funded in this context, introducing the missing complementary approach and innovative solutions which may be applied in similar localities as well as for big cities.

\section{The Safely Connected Project: Implementation and Main Results}

The main results concern the definition of basic principles for the design activity pursued in the framework of the Safely connected initiative (Decalogue), and the practical solutions for their in-field implementation (Practical initiatives).

The decalogue consists of a series of architectural and urban planning strategies for public space design. On the one hand, these are promoting and protecting public health, well-being, safety \& security, and on the other, they are improving the quality of the commercial urban district involving retailers as "experience directors" and giving consumers the opportunity to take on an increasingly active role in the enjoyment, and in some cases co-creation, of their own consumer experience.

The Practical initiatives concern the creation of flexible physical and digital tools for the safe resumption of the economy and urban life and generating a shift in favour of the sustainable use of public spaces. The aim was to create an urban microcosm designed to reconfigure the physical and social space Fig. 3, composed of several parts with different functions that, thanks to its shapes and geometries, ensures compliance with anti-Covid standards, allows potential users to customize and complete it, contributes to the quality of space and social interaction and is useful for various types of user, including retailers.

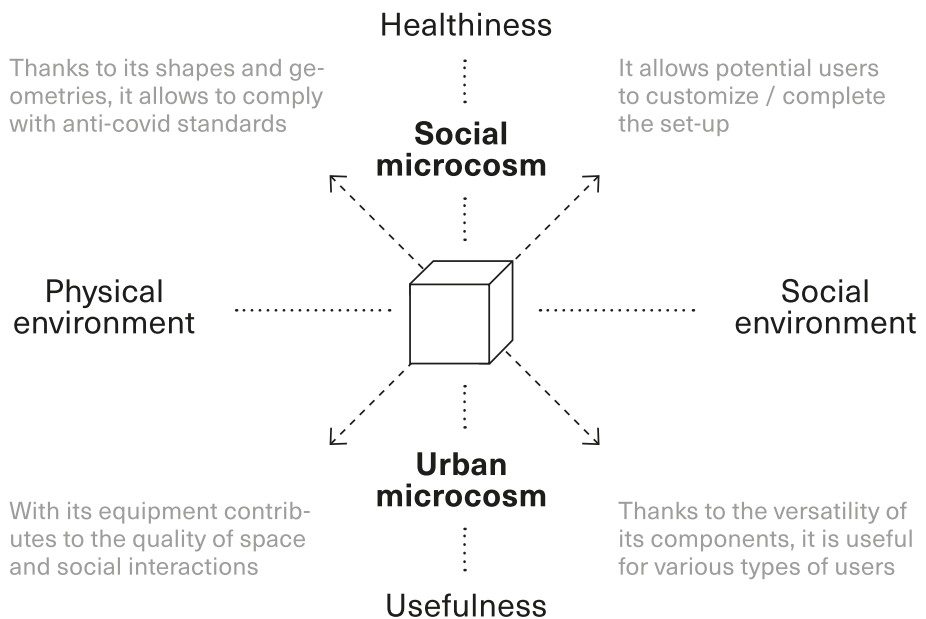

Fig. 3

Conceptualisation of the solution. 
The different combinations of elements are designed to colonize and increase the functionality of the public space, enrich the experience of citizens and visitors and create a lively pedestrian environment that people can enjoy and where they can develop a sense of belonging thanks to the perception of higher quality. The quality is felt, not as a result of a bespoke outstanding appearance, but of the enhanced and increased utilisation. A substantial move away from a formal connotation of design, in favour of uses and performance.

The result is also a new approach towards the existing built heritage. Based on simple geometry inspired by ancient decorations, the new system has a lightweight transparent appearance Fig. 4 which does not obstruct the perception of the historical urban landscape. However, a different version is also included: a stronger formal and playful language offering more complex and customised installations to be applied in the more modern districts of the city Fig. 5 .

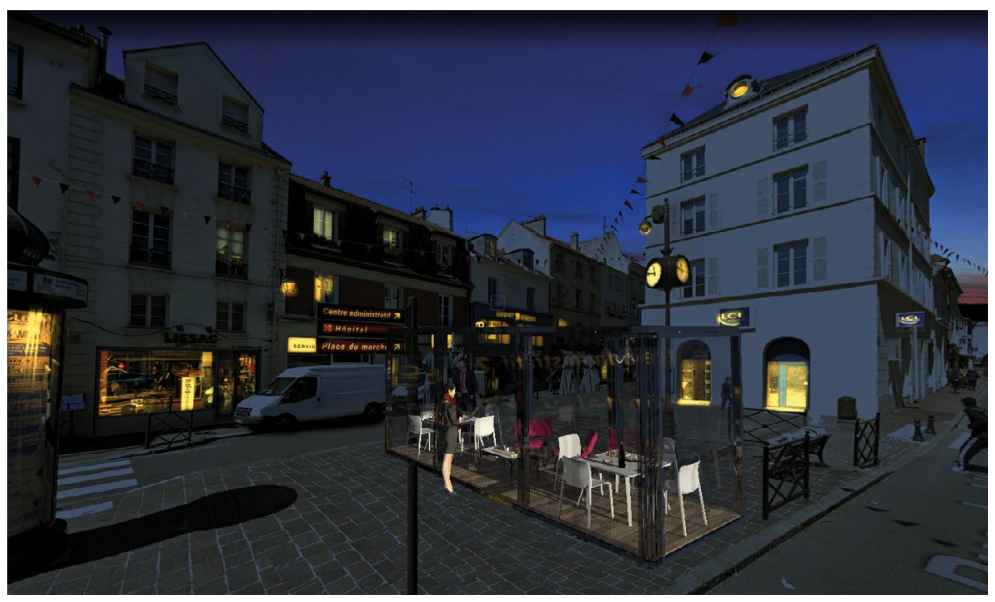

Fig. 4

Rendered image of the design solution.

Fig. 5

The parklet system alternative configu ations by $\mathrm{R}$. Trocchianesi and $\mathrm{C}$. Piantanida, Department of Design, Politecnico di Milano). 
The digital part of the system goes far beyond a classical e-commerce platform Fig. 6, fostering the solidarity of inhabitants and retailers, as well as their sense of community, while encouraging active modes of transport and supporting the revival of cultural and sports activities. Conceived on the basis of input from retailers regarding their needs and correlated with the rethinking of the use of various areas and traffic flows on the test site, the platform aims to support both the revival of people-friendly lively public spaces, and the economic relaunch of old city centres. Furthermore, it represents an essential component of the people-centred approach of Safely Connected, backing the sense of community and the creation of a solidarity network much needed during times of crisis. This tailor-made innovative digital tool resembles a series of advanced services (for local businesses and their clients) enabling the optimisation of business models and the creation of new business opportunities.

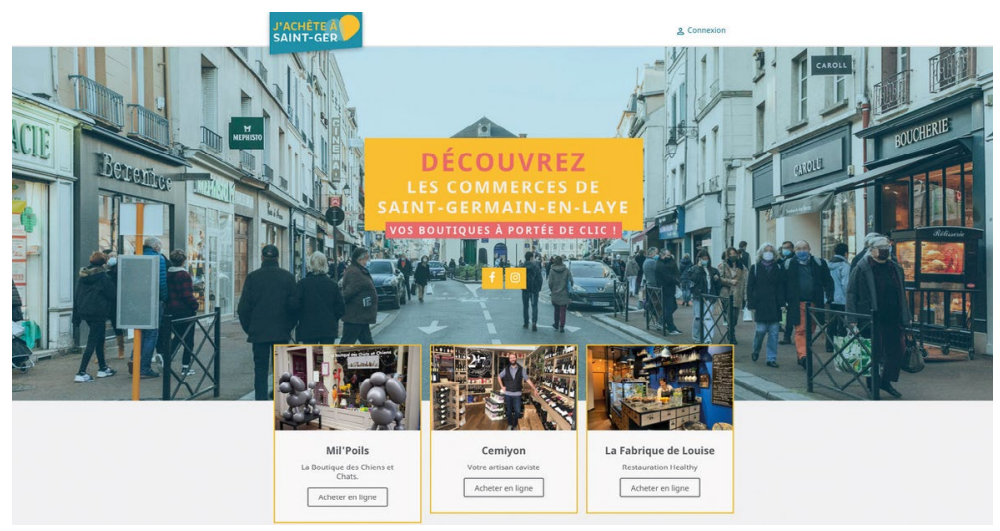

The development of new personal skills, with the help of self-learning paths, generates innovation and allows the system to evolve, improving the proposed solutions or formulating new hypotheses.

The project achieved significant results, in spite of the constraining, and continuously changing lockdown restrictions which also imposed several changes to its implementation.

The interviews and focus groups with local businesses were complemented by a new telephone survey with the residents, regarding commercial activity and the appreciation and use of public spaces in the pilot area. This investigation served to define the baseline before the implementation of the solutions and to set detailed goals, key performance indicators and targets. Several rounds of online and offline consultations with local inhabitants, businesses and politicians were organised to optimise, test, and validate the proposed solutions for public space configurations, the urban furniture, and the e-commerce platform. These frequent exchanges also helped mitigate unforeseen difficulties and strengthen the content of the project through the introduction of new features such as the addition of an urban logistics platform. Created at the periphery of the city-centre, this enabled sustainable and shared deliveries for city centre businesses as well as grouped
Fig. 6 A preview of some of the shops listed under the 'Home' category. 
deliveries to their clients over an extended area. Thus, it supported the extension of the pedestrian area without challenging the local businesses already severely affected by the sanitary crisis, as well as significantly reducing carbon emissions in the city centre.

\section{Conclusions}

The EIT Project represented for SGL and its partners an opportunity for innovation and to test novel approaches impossible to adopt under the previous normal circumstances. It forced the public administration to review its scheduling and modes of operation, favouring cross-sector and multilevel cooperation and the Politecnico di Milano team to implement and simultaneously enhance the skills and competences developed.

Despite the adversities, the distinctive competences and the multidisciplinary features of the co-opted partners helped to collaboratively enrich the general vision and effectively adapt the specific actions so as to better respond to any unexpected events and capitalise on new opportunities.

In a quite uncommon way, this situation transformed the implementation of the project into a highly meaningful and unique learning experience, at the same time augmenting the value and usability of the deliverables, proving their applicability and performance in an extremely difficult context. Accordingly, the project is an outstanding example of "shelf innovation" (Celaschi, 2015), that was possible thanks to the ongoing academic research targeting co-design methodologies, the quality and safety of public spaces, and the quest for sustainability goals in open spaces, enhanced and implemented through the Safely Connected project following a multidisciplinary collaborative approach.

The co-designer methodologies were interpreted in the different stages of the research: the brief to investigate and integrate both inhabitants and stakeholders needs as well as the requests of the Municipality, the improvement of the proposal through intermediate exchanges and the final evaluation. Moreover, the concept embeds a co-design idea developing over time, not only because the transformation is conceived as a process through different phases of extension to the city centre, but also because the new infrastructures are intended as an open system. The installations to furnish the renovated SGL outdoor spaces were designed with flexibility in mind, offering not only multiple possible layouts, but also customisation in terms of mood and identity as well as of functions, using, re-using and recycling the same components thanks to the easy assembly and construction. Finally, the health issue was also interpreted through customisation, allowing for different changeable layouts to comply with the physical distancing recommendations. 


\section{Interview Box}

Stefano Capolongo is the principal investigator of the EIT Urban Mobility "Safely Connected" research project.

Which topics does the Safely Connected project address?

SC The project rests on the idea that Public Health is not only about protection and promotion, but it is also an individual and a collective condition, strongly influenced by the environmental context and by the strategies implemented by local governments. Both the link between morphological and functional characteristics of urban contexts and the impacts of Public Health open up a new technical and design scenario on Urban Health; this has been approached resting upon the ability to systematize:

- $\quad$ health impacts: mostly represented by the main chronic-degenerative diseases (NCD's), whose onset derives from the quality of the urban environment and the adoption of unhealthy lifestyles;

- environmental risk factors (urban heat island, atmospheric acoustic and visual soil pollution, vehicular traffic, safety and protection and inadequate places appeal);

- healthy urban planning strategies (green/blue/ grey infrastructures planning, biodiversity protection problem, adverse and sudden weather event management problem, intermodal public transport system planning, reduction of vehicular traffic problem, cycling and pedestrian routes planning, social and functional mixite, municipal solid waste management, efficiency optimization of renewable energy systems, the open space lighting problem and the Design for All strategy).

Moreover, Safely Connected aims at health promotion in urban planning based on the definition and application of Health Urban Planning and Design Strategies, which are the most effective in the creation of an urban environment, both indoor and outdoor, and which are able to promote indoor well-being and encourage the adoption of healthy lifestyles.

Which are the approach and the methodologies adopted?

SC Urbanisation and the layout of contemporary cities cause as many risks to public and individual health as opportunities. In the cross-sectoral policies' field, the "Health in All Policies" strategy clearly states that health depends not only on the distribution of health services and lifestyles, but also on the quality of living and working environments, the perspective of economic situation improvement, on community cohesion and on availability of high-quality public services. Many of these aspects are conditioned by proper planning, designing and management of urban spaces; the challenge is to create a correct and conscious design for outdoor and indoor environments. The scientific 
community also hopes that protocols and certifications about "Healthy Environment" will be adopted, similar to those already existing and widespread on sustainability of buildings and neighbourhoods.

What is the scientific background of the research?

SC Inter-sectoral research, together with the scientific activity of independent companies - such as the Urban Health Section of the European Public Health Association (EUPHA) support cultural progresses in this direction. In March 2017 we promoted, at the High School of Epidemiology and Preventive Medicine "Giuseppe D'Alessandro" in Erice - Ettore Majorana Foundation and Centre for Scientific Culture - the "Strategies for Disease Prevention and Health Promotion in Urban Areas" course, which involved over 60 academics including Architects, Urban planners, Epidemiologists and Trainees in Hygiene and Preventive Medicine. In March 2019 we hosted, at Politecnico di Milano, the 1st European Symposium "Salutogenic Hospital Design \& Urban Health" of the International Academy Design \& Health (IADH). In March 2021 we are planning, at the Erice School, the "re-Thinking, Designing and Managing Salutogenic Cities" course.

Which are the future challenges imposed by the current pandemic crisis?

SC COVID-19 pandemic is an important demonstration of the double effects of urbanization on the environment: the inherent capacity of contemporary cities to be places for social and economic opportunities and simultaneously containers of multiple risk factors for Public Health and Sanitary Welfare. The sudden lifestyles change, due to physical distancing, is pressuring the transformation of cities into resilient ecosystems capable of promoting health and preventing the spread of today's and tomorrow's infectious diseases. The challenge is to restore the dialogue between technical professionals and public health experts. It is necessary to undertake a multidisciplinary approach among Designers (Urban planners and Architects), Policy Makers (Public Administrators), Public Health Experts (Epidemiologists, Health Local Agencies, etc.) and citizenship (City Users, Associations, etc.).
Laura Daglio

Laura Daglio is Associate

Professor in Architectural Technology at Politecnico di Milano. She is interested in regeneration of the built environment and has developed co-design and meta-design approaches for participated projects aimed at the bottom-up involvement of communities in the design phase.

\section{Daniele Fanzini}

Daniele Fanzini, is

Associate Professor in Architectural Technology at Politecnico di Milano. He is interested in urban regeneration practiced through the Project Anticipation approach and participation. $\mathrm{He}$ has managed several projects with wide involvement of public and private organisations.

\section{Elena Mussinelli}

Elena Mussinelli is Full Professor in Architectural Technology at Politecnico di Milano. She is interested in environmental design with a particular regard to the innovation dynamics within the production of the project and the updates of the legislative framework in the field of environmental quality.

\section{Irina Rotaru}

Irina Rotaru is an independent researcher and European expert, Ph.D. in Design and Technologies for the Enhancement of Cultural Heritage. Focused on the co-design strategies for urban regeneration and cultural design, she works as a project manger of the city of SGL, trainer, cultural manager, and editor for Zeppelin magazine. 


\section{References}

Battisti, A., Mussinelli, E., \& Rigillo, M. (2020). Public space and urban quality. Techne, Journal of Technology for Architecture and Environment, 19, 17-23. https://doi.org/10.13128/ techne-7933

Boeri, A., Fini, G., Gaspari, J., Gianfrate, V., \& Longo, D. (2018). Bologna resilient city: from the adaptation plan to local actions. Techne, Journal of Technology of Architecture and Environment, 15, 193-202. https://doi.org/10.13128/ Techne-22103

Camocini, B., Daglio, L., Gerosa, G., \& Ragazzo, S. (2020). Projects for the temporary reactivation of public space: what legacy? Techne, Journal of Technology of Architecture and Environment, 19, 125-133. https://doi.org/10.13128/ techne-7828
Casoni G., \& Fanzini D. (2011). I luoghi dell'innovazione. Maggioli.

Celaschi, F. (2015). Advance Design Points of View". In Celi, M. (ed.), Advanced Design Cultures. Long-Term Perspective and Continuous Innovation (pp. 3-18). Springer.

Celaschi, F., Formia, E., \& Vai, E. (2019). Mutating City: Designing Events as a Matter of Social Innovation. Strategic Design Research Journal, 12(3), 323-337. http://dx.doi.org/10.4013/ sdrj.2019.123.03

GDCI \& Nacto (2016). Global Street Design Guide. Washington DC Island Press. https://globaldesigningcities.org/publication/ global-street-design-guide/
Freire, K., Del Gadio, C., \& Franzato C. (2017)

Design-driven strategies for creative social innovation ecosystem, International Journal of Knowledge Engineering and Management, 6 (16), 46-49. https://www. academia.edu/26431659/ Design_driven_strategies_ for_creative_social_innovation_ecosystems

Gianfrate, V., \& Longo, D. (2017). Urban micro-design Tecnologie integrate, adattabilità e qualità degli spazi pubblici. Franco Angeli.

Mallach, A. (2006). Bringing Buildings Back. From abandoned Properties to Community Assets. Rutgers University Press.

Manzini, E. (2015). Design when everybody designs. The MIT Press.

Mussinelli, E. (2018). II progetto ambientale dello spazio pubblico. Ecowebtown, 18. http://www. ecowebtown.it/n_18/.
Project for Public Spaces (2018). Placemaking. What if we built our cities around places? https://www.pps. org

Losasso, M. (2017). Progettazione ambientale e progetto urbano, Ecowebtown, 16. http://www.ecowebtown.it/n_16/

Schiaffonati, F. (2016). Paesaggio italiano. Viaggio nel paese che dimentica, Lupetti.

Teli, M., Bordin, S., Menendez Blanco, M., Orabona G., \& De Angeli A. (2015), Public design of digital commons in urban places: A case study. International Journal of Human-Computer Studies, 81, 17-30. https://doi.org/10.1016/j. ijhcs.2015.02.003 